\title{
Infrared Astronomy: In the Heat of the Night The 1999 Ellery Lecture
}

\author{
John W. V. Storey \\ School of Physics, University of New South Wales, NSW 2052, Australia \\ j.storey@unsw.edu.au \\ Received 2000 July 10, accepted 2000 August 3
}

\begin{abstract}
Infrared astronomy has made remarkable progress over the past quarter century. This paper, which is a brief summary of the 1999 Ellery Lecture, outlines the importance of the infrared, traces some of the key Australian developments in the field, and looks ahead to the challenges facing infrared astronomers in the immediate future.
\end{abstract}

Keywords: infrared—history

\section{The Infrared Advantage}

It was exactly 200 years ago, in 1799 , that William Herschel proffered the somewhat tentative suggestion: 'Radiant heat will at least partly, if not chiefly, consist, if I may be permitted the expression, of invisible light.' (See, for example, King 1955.) The year 1999 is therefore a particularly appropriate one in which to review the current state of infrared astronomy.

The past 25 years have witnessed an extraordinary transformation in infrared astronomy, as it moved from being the preserve of a few physicists to become a mainstream activity at virtually every 'optical' observatory around the world. Almost no new 'optical' telescope is designed now without a major effort going into optimising its infrared performance. The so-called Next Generation Space Telescope (NGST) will probably not work in the optical at all, being purely an infrared telescope.

To the astronomer, the infrared region offers substantial advantages. Some of the most important of these are:

- Lower extinction. Interstellar extinction is a very steep function of wavelength. Heavily obscured regions, such as the centres of galaxies and starformation regions, cannot be observed at optical wavelengths but are readily studied in the infrared.

- The ability to see warm (as opposed to hot) objects. Planets, circumstellar discs, protostars and other warm objects emit negligible flux at visible wavelengths, and can therefore only be directly observed at infrared and longer wavelengths.

- Better spatial resolution under seeing-limited conditions. The diameter of an image under seeing-limited conditions goes as $\lambda^{-1 / 5}$. In addition, adaptive optics correction becomes much simpler and much more effective as the wavelength increases.

- Unique phenomena. In any spectral region there will be phenomena that can be observed at other wavelengths only with difficulty, or perhaps not at all. In the infrared region, for example, lie almost all of the molecular vibrational transitions, plus the rotational lines of molecular hydrogen and HD.
- Red-shifted spectral features. Important diagnostic lines whose rest-frame wavelengths are in the optical or ultraviolet are shifted into the infrared at high $z$.

Of course, there are also substantial difficulties to be overcome when working in the infrared. To begin with, the atmosphere is opaque except for a few wavelength bands or 'windows'. Strong, saturated absorption lines of molecules such as water, carbon dioxide and ozone are present at intervals across the spectrum, leaving many wavelength regions unobservable from the ground.

Secondly, the sky is raining photons. Beyond about $2.2 \mu \mathrm{m}$, anything at room temperature and above is emitting copious amounts of black-body radiation. For the infrared astronomer it is as if the telescope were brightly illuminated and the sky itself glowing. Not only does the flood of photons create a high backgroundlimited noise floor, but the sheer number of photons (up to $10^{10}$ per second per pixel) presents a significant technological challenge.

Finally, and partly as a result of the last two considerations, the technology needed to work effectively in the infrared has lagged that of the optical region by a decade or more.

\section{Twenty-five Years of Australian Infrared}

This section is not intended to be an exhaustive history of Australian infrared astronomy, but rather to illustrate some of the major advances that have shaped our present knowledge and expertise. These advances have, of course, been driven by the development of ever more capable instrumentation.

\subsection{Near Infrared}

In 1971, Harry Hyland noted in an article in PASA (Hyland 1971) that virtually all infrared observations conducted to that time had been undertaken in the northern hemisphere, and correctly predicted that '. . . the Southern Hemisphere is still a virtually untapped reservoir, and offers wide scope for major advances in the field.' For the next few years, Harry and his colleagues at Mount Stromlo Observatory and 
elsewhere used a single-pixel bolometer instrument to make the first forays into this 'untapped reservoir'.

This infrared photometer was progressively upgraded, moving from the initial bolometer system for observations from 2-20 $\mu \mathrm{m}$, through a $\mathrm{PbS}$ system for short-wavelength photometry, to a modern, highsensitivity 1-5 $\mu \mathrm{m} \mathrm{InSb}$ system. This was one of the first fully automated and computer-controlled infrared photometers constructed, and led, among other things, to the discovery of young stars in the LMC.

In 1978 the Infrared Photometer/Spectrometer (IRPS) was developed at the Anglo-Australian Observatory (AAO) by David Allen and the AAO engineers (Barton \& Allen 1987). The IRPS represented a huge leap forward in 'user friendliness'. No longer was it necessary for the observer to understand all the intricacies of the infrared detection process. With its digital integration technology and simple menu-driven software, the IRPS was the first truly civilised infrared instrument available to astronomers anywhere in the world.

The IRPS thus brought infrared astronomy into the mainstream. Its discoveries included:

- images of the surface of Venus

- molecular hydrogen in many planetary nebulae

- polarimetric studies

- complex structure of the Galactic Centre region.

The IRPS was finally decommissioned in 1994, after nearly two decades of exceptionally productive work on the Anglo-Australian Telescope (AAT). Even then it refused to give up. In 1994 it was loaned to the University of New South Wales, who 'winterised' it and installed it at the South Pole for site-testing studies (Ashley et al. 1995). When its work was completed there, it was returned to the AAO where, at the time of writing, it is still being used-this time as a testbed for cryogenic infrared fibres!

In 1978, Terry Jones and Harry Hyland designed a cooled-grating spectrometer (CIGS) which employed novel cylindrical optics to allow large beam observations at intermediate resolving power $(\sim 1000)$, while nevertheless fitting within a conventionally sized dewar (Jones et al. 1982). This instrument proved extremely powerful in observations of extended sources, and in obtaining very high signal/noise data of sources such as SN1987A.

A modified version of this spectrometer was designed for the AAT, where it was known as FIGS, the Fabry-Perot Infrared Grating Spectrometer (Bailey et al. 1988). (The 'Fabry-Perot' part referred to an ingenious but ultimately ill-fated plan to use a thin silicon wedge in front of the entrance window as a tunable FabryPerot.) FIGS used cylindrical optics and an array of 16 discrete detectors. FIGS was available on the AAT from 1985 to 1991 . Perhaps its best known scientific result was its observation of the evolution of SN1987A, in which it was able to detect a vast array of atomic and ionic species, plus $\mathrm{CO}, \mathrm{CO}^{+}$and possibly CS (Meikle et al. 1989).

In the early eighties, although two-dimensional monolithic detector arrays were starting to find their way into the hands of US astronomers, strict military controls prevented their export to even friendly nations such as Australia. This prompted the University of New South Wales to embark on an ambitious program in 1982 to fabricate monolithic infrared detector arrays based on platinum silicide technology (e.g. Kurianski, Green \& Storey 1986). A $32 \times 4$ pixel device was ultimately tested on the AAT; it was able to detect Sirius through thick clouds but was never used for any serious astronomical research.

In the meantime, export controls were beginning to relax and the Anglo-Australian Telescope was able to obtain one of the early 'NICMOS' detectors (so-called because their development was funded not through defence channels but for use on the NICMOS instrument of the Hubble Space Telescope). This detector is of a hybrid type, where the detector material (in this case mercury cadmium telluride) is bump-bonded via indium balls onto a silicon multiplexer. With $128 \times 128$ pixels, the NICMOS detector represented a major advance. It was built into IRIS, a versatile infrared camera/spectrometer designed once again by David Allen and the AAO engineers (Gillingham \& Lankshear 1990; Allen et al. 1993; Gillingham 1993a). IRIS was a technological tour de force, winning the prestigious Bradfield Award of the Sydney Division of the Institution of Engineers, Australia in 1993.

For the past decade IRIS has made a major contribution to southern hemisphere astronomy. One of its images_-of the molecular hydrogen and [FeII] 'bullets' in Orion-even appeared on the front cover of Nature (Allen \& Burton 1993).

IRIS was subsequently enhanced by the addition of a polarimetry module (Hough, Chrysostomou \& Bailey 1994; Gledhill, Chrysostomou \& Hough 1996) and a Fabry-Perot (UNSWIRF ${ }^{1}$; Ryder et al. 1998). Perhaps David Allen's final legacy to infrared instrumentation in Australia was to set in motion the construction of a successor to IRIS. IRIS-II (a wide-field infrared camera/ spectrometer with a $1024 \times 1024$ pixel detector) is currently under construction at the AAT and due for commissioning on the telescope in 2000/2001 (Gillingham \& Jones 2000).

In the late 1990s the ' $3 \mathrm{D}$ ' instrument of the Max Planck Institut für Extraterrestrische Physik (MPE) was made available on the AAT. With the ability to produce 256 simultaneous spectra over a $16 \times 16$ pixel region of the sky, 3D was the first of a new generation of 'integral field' spectrometers (Weitzel et al. 1996). Its success was largely responsible for the current resurgence of interest in integral field techniques.

1 Named after the then premier of New South Wales 
Shortly after IRIS was built, Peter McGregor and the MSSSO engineers developed CASPIR for the $2.3 \mathrm{~m}$ telescope on Siding Spring (McGregor et al. 1994). Sporting a $256 \times 256$ indium antimonide array, CASPIR was able to operate out to $5.6 \mu \mathrm{m}$ (compared to IRIS's limit of $2.5 \mu \mathrm{m}$ ). Amongst CASPIR's best-known results were a spectacular set of images of the impact of Comet Shoemaker-Levy 9 on Jupiter in July 1994 (McGregor, Nicholson \& Allen 1996). CASPIR is currently the only instrument in Australia covering this wavelength range, and continues to produce excellent science.

In parallel with the instrumentation development of the past quarter century was another crucial area that required painstaking attention before near-infrared astronomy could become routine: precision photometry of standard stars. Early work on JHKL standards (Jones \& Hyland 1982; Allen \& Cragg 1983) allowed the field to get under way and tied the southern hemisphere standards to those of the north (Elias et al. 1983), work which was further refined by McGregor (1994) and Carter \& Meadows (1995).

\subsection{Mid Infrared}

The early days of mid-infrared astronomy were pioneered by Harry Hyland (at MSSSO) and John Thomas (at the Royal Australian Air Force Academy, then part of the University of Melbourne) using single-element bolometer systems (e.g. Robinson, Hyland \& Thomas 1973; Thomas, Hyland \& Robinson 1973). The field received an enormous boost in 1978 with the arrival at the Anglo-Australian Observatory of David Aitken and Patrick Roche, who brought with them the 'UCL' midinfrared grating spectrometer (Aitken et al. 1979). This simple but remarkably effective instrument used an array of five discrete detectors (later upgraded to 16 and then to 30), and dominated the field of mid-infrared spectroscopy for the next decade. It remained the only mid-infrared instrument available in Australia until the early nineties, when MIRAS/NIMPOL was commissioned on the AAT by Craig Smith (Smith et al. 1997), followed by MANIAC, developed by the UNSW group in collaboration with the MPE (Boeker et al. 1997).

\subsection{Far Infrared}

Blocked by water vapour in the Earth's atmosphere, farinfrared radiation can only be observed from space, balloon or aircraft platforms. In the mid-seventies John Thomas carried out Australia's first pioneering work in this region with a balloon-borne telescope (Thomas 1977). In 1977, the Kuiper Airborne Observatory, a NASA-operated C-141 Starlifter fitted with a $90 \mathrm{~cm}$ telescope (Cameron, Bader \& Mobley 1971), visited Australia to observe the occultation by Uranus of a background star (an observation which, incidentally, first detected the rings of Uranus; Elliot, Dunham \& Mink 1977). The success of this visit led to considerable enthusiasm in Australia for a formal program of expeditions to be established (Storey 1982). In 1983, the Kuiper Airborne Observatory returned to fly a series of missions from Richmond Airforce Base in NSW. Once again a block of time was set aside for Australian astronomers, this time to fly six flights as principal investigators.

Just when it appeared that these collaborations would grow to the point where the Kuiper Airborne Observatory would become a regular visitor to Richmond, political machinations within the RAAF forced the cancellation of the 1987 visit (which was planned to coincide with the return of Comet Halley). At the last moment, NASA was forced to shift its operations to Christchurch, New Zealand. The Kuiper Airborne Observatory has made regular visits there ever since, until its recent decommissioning to make way for SOFIA.

\section{New Developments}

\subsection{Big Telescopes}

For the past quarter century the $4 \mathrm{~m}$ telescope has been the ultimate tool for ground-based astronomical research. This is no longer the case. There are now a number of 8 and $10 \mathrm{~m}$ telescopes in operation, and even more under construction. In the infrared, where the image size can be that of the entire diffraction-limited telescope aperture (using adaptive optics in the near infrared, or simply taking advantage of good seeing conditions in the mid infrared), the point-source sensitivity goes as the square of the telescope diameter. The advantage of a large telescope in the infrared is thus substantially greater than it is in the visible, where sensitivity usually improves only linearly with telescope diameter. Furthermore, the new generation of large telescopes are being built on superb infrared sites, and the telescopes themselves (especially Gemini) are being optimised for extremely low emissivity in the infrared. Unfortunately, all of these factors argue against the future construction of a large optical/infrared telescope in Australia. However, by becoming a 5\% partner in the Gemini project, Australia has been able to maintain access to some of the best facilities in the world, an essential prerequisite for the continued health of the country's astronomy program.

Australia has also been able to capitalise on its strengths in infrared instrumentation within the Gemini partnership, recently signing a contract for the fabrication of a near-infrared integral field spectrograph (NIFS) for Gemini North. To be built at Mount Stromlo Observatory, NIFS is designed to make full use of the high-resolution capabilities of the adaptive optics facility of the telescope.

\subsection{Air and Space}

In the US, two major projects are about to come to fruition. The first, SIRTF (the Space Infrared Telescope 
Facility) is due to be launched in December 2001 (Bicay et al. 1998). SIRTF will be placed into an Earth-trailing heliocentric orbit, and will observe from 3 to $180 \mu \mathrm{m}$. It will have cryogenic optics, and a primary mirror diameter of $85 \mathrm{~cm}$. The second major aerospace project, SOFIA (the Stratospheric Observatory for Infrared Astronomy), is the successor to the Kuiper Airborne Observatory (see, for example, Erickson \& Davidson 1995; Becklin 1997). A joint venture of NASA and the German DLR, SOFIA will carry a $2.5 \mathrm{~m}$ telescope to a flight altitude of $12.5 \mathrm{~km}$ and will observe from $0.3 \mu \mathrm{m}$ to $1.6 \mathrm{~mm}$. Its first science flights are scheduled for 2001.

\subsection{Interferometry}

While two-telescope interferometry in the infrared has been a reality for the past quarter century, the next few years will see an explosion in the growth of this field. Both heterodyne interferometry (e.g. Lipman et al. 2000) and direct-detection methods (e.g. Koresko et al. 1998; Young et al. 1998; Mennesson et al. 1999) have now reached high levels of maturity. The very high spatial resolutions obtainable promise the detection of circumstellar discs and Jupiter-like planets around other stars, and the opportunity to explore deep into the cores of active galactic nuclei.

\subsection{Antarctica}

For ground-based telescopes in the thermal infrared, background radiation from the atmosphere and the telescope remains the fundamental limit to achievable sensitivities. By going to the Antarctic Plateau, where ambient temperatures fall to $-80^{\circ} \mathrm{C}$ and the sky is exceptionally dry, substantial improvements in performance can be expected. There are also strong indications that the seeing from high-altitude Antarctic sites is better than from anywhere else on the Earth's surface (Gillingham 1993b). To date, all of the infrared site-testing work in Antarctica has been carried out at the AmundsenScott station at the South Pole (Ashley et al. 1996; Nguyen et al. 1996; Smith \& Harper 1998; Phillips et al. 1999; Chamberlain et al. 2000). The results there show a 10 - to 50-fold decrease in sky brightness across the infrared, implying that very large gains in the sensitivity could be achieved.

Of all the nations currently involved in astronomy, Australia stands to benefit the most from the deployment of a medium-sized infrared telescope on the Antarctic Plateau. Not only does Australia have a tradition of Antarctic exploration, but it also has a major, ongoing national Antarctic program. Furthermore, there are no observing sites in Australia that can compare with those in Chile or Hawaii, and Australia is actually closer to Antarctica than it is to those sites anyway!

With these considerations in mind, Australia is currently leading the push to deploy a medium-sized ( $2 \mathrm{~m}$ ) infrared telescope on the Antarctic Plateau. Such a telescope could not only perform unique science in its own right, it could also act as a 'finder' telescope of unsurpassed capability for Gemini and perhaps the NGST.

\section{Concluding Remarks}

Since the first humans looked up at the stars and, in so doing, unwittingly became the first astronomers, our perception of the universe has been coloured by our own biological limitations. With eyes that respond only to a very narrow range of wavelengths (roughly 400-700 nm), we have striven first to see fainter and fainter objects with ever-increasing clarity. Only in the past few years have we been able to obtain images in other parts of the spectrum that 'look' as they would if our eyes worked at those wavelengths. First came aperture synthesis radio astronomy, then infrared imaging techniques using million-pixel detectors and then, more recently, high-resolution X-ray images. Our eyes have now had their wavelength response extended to cover most of the electromagnetic spectrum. For the first time in our evolution as a species, we can 'look' at the Universe without any predetermined wavelength bias, and see it as it really is.

Australia's contribution to this new view of the universe has been substantial. As we enter the third century of infrared astronomy, we can only wonder what new discoveries are in store, and what changes they will bring to our perception of the world.

\section{Acknowledgments}

The Ellery Lecture is held every two years in memory of Robert Ellery. Ellery was Director of Melbourne Observatory for much of the second half of the 19th century, and one of the outstanding workers in Australian astronomy in that era. I thank Mike Bessell, Peter Gillingham, Harry Hyland, Peter McGregor and the two anonymous referees for their helpful suggestions, all of which have helped improve this paper.

\section{References}

Aitken, D. K., Roche, P. F., Spenser, P. M., \& Jones, B. 1979, A\&A, 76, 60

Allen, D. A., et al. 1993, PASA, 10, 298

Allen, D. A., \& Burton, M. G. 1993, Nature, 363, 54

Allen, D. A., \& Cragg, T. A. 1983, MNRAS, 203, 777

Ashley, M. C. B., Burton, M. G., Lloyd, J. P., \& Storey, J. W. V. 1995, Proc. SPIE, 2552, 33

Ashley, M. C. B., et al. 1996, PASP, 108, 721

Bailey, J., et al. 1988, PASP, 100, 1178

Barton, J. R., \& Allen, D. A. 1987, PASP, 92, 368

Becklin, E. E. 1997, in Proc. ESA Symposium 'The Far Infrared And Submillimetre Universe', 15-17 April 1997, Grenoble, France, ESA SP-401 (Noordwijk: ESA), p. 201

Bicay, M. D., Werner, M. W., Latter, W. B., \& Simmons, L. L. 1998, Proc. SPIE, 3356, 653

Boeker, T., Storey, J. W. V., Krabbe, A., \& Lehmann, T. 1997, PASP, 109, 827 
Cameron, R. M., Bader, M., \& Mobley, R. E. 1971, Appl. Opt., 10,2001

Carter, B. S., \& Meadows, V. S. 1995, MNRAS, 276, 734

Chamberlain, M. A., Ashley, M. C. B., Burton, M. G., Phillips, A., Storey, J. W. V., \& Harper, D. A. 2000, ApJ, 535, 501

Elias, J. H., Frogel, J. A., Hyland, A. R., \& Jones, T. J. 1983, AJ, 88,1027

Elliot, J. L., Dunham, E., \& Mink, D. 1977, Nature, 267, 328

Erickson, E. F., \& Davidson, J. A. 1995, in Airborne Astronomy Symposium on the Galactic Ecosystem: From Gas to Stars to Dust, vol. 73, ed. M. R. Haas, J. A. Davidson \& E. F. Erickson (San Francisco: ASP), p. 707

Gillingham, P. R. 1993a, in Optics in Astronomy, ed. J. V. Wall (Cambridge Univ. Press), p. 39

Gillingham, P. R. 1993b, in Optics in Astronomy, ed. J. V. Wall (Cambridge Univ. Press), p. 244

Gillingham, P., \& Jones, D. 2000, Proc. SPIE, 4008, 1084

Gillingham, P. R., \& Lankshear, A. F. 1990, Proc. SPIE, 1235, 9

Gledhill, T. M., Chrysostomou, A., \& Hough, J. H. 1996, MNRAS, 282, 1418

Hough, J. H., Chrysostomou, A. C., \& Bailey, J. A. 1994, in Infrared Astronomy with Arrays: the Next Generation, ed. I. S. McLean (Dordrecht: Kluwer), p. 287

Hyland, A. R. 1971, PASA, 2, 14

Jones, T. J., \& Hyland, A. R. 1982, MNRAS, 200, 509

Jones, T. J., Hyland, A. R., Dopita, M. A., Hart, J., Conroy P., \& Hillier, J. 1982, PASP, 94, 207

King, H. C. 1955 (republished 1979), The History of the Telescope (New York: Dover)
Koresko, C. D., et al. 1998, ApJ Lett., 509, L45

Kurianski, J. M., Green, M. A., \& Storey, J. W. V. 1986, IEEE Electron. Device Lett., 7, 140

Lipman, E. A., Hale, D. D. S., Monnier, J. D., Tuthill, P. G., Danchi, W. C., \& Townes, C. H. 2000, ApJ, 532, 467

McGregor, P. 1994, PASP, 106, 508

McGregor, P., Hart, J., Downing, M., Hoadley, D., \& Bloxham, G. 1994, Experim. Astron., 3, 139

McGregor, P. J., Nicholson, P. D., \& Allen, M. G. 1996, Icarus, 121,361

Meikle, W. P. S., Spyromilio, J., Varani, G.-F., \& Allen, D. A. 1989, MNRAS, 238, 193

Mennesson, B., et al. 1999, A\&A, 346, 181

Nguyen, H. T., et al. 1996, PASP, 108, 718

Phillips, A., et al. 1999, ApJ, 527, 1009

Robinson, G., Hyland, A. R., \& Thomas, J. A. 1973, MNRAS, 161,281

Ryder, S. D., Sun, Y.-S., Ashley, M. C. B., Burton, M. G., Allen, L. E., \& Storey, J. W. V. 1998, PASA, 15, 228

Smith, C. H., Aitken, D. K., Moore, T. J. T., \& Fujiyoshi, T. 1997, PASA, 14,179

Smith, C. H., \& Harper, D. A. 1998, PASP, 110, 747

Storey, J. W. V. 1982, PASA, 4, 474

Thomas, J. A. 1977, PASA, 3, 97

Thomas, J. A., Hyland, A. R., \& Robinson, G. 1973, MNRAS, 165,201

Weitzel, L., et al. 1996, A\&AS, 119, 531

Young, J. S., et al. 1998, Proc. SPIE, 3350, 746 